This is the peer reviewed version of the following article: McCallion P, Hogan M, Santos FH, et al. Consensus Statement of the International Summit on Intellectual Disability and Dementia Related to End-of-life Care in Advanced Dementia. Journal of Applied Research in Intellectual Disabilities 2017;30:1160-1164, which has been published in final form at https://doi.org/10.1111/jar.12349. This article may be used for non-commercial purposes in accordance With Wiley Terms and Conditions for self-archiving. 


\section{Consensus Statement of the International Summit on Intellectual Disability and Dementia Related to End-of-life Care in Advanced Dementia ${ }^{1}$}

McCallion, P. ${ }^{1}$, Hogan, M. ${ }^{2}$, Santos, F H. ${ }^{3}$, McCarron, M. ${ }^{4}$, Service, K. ${ }^{5}$, Stemp, S. ${ }^{6}$, Keller, S. ${ }^{7}$, Fortea, J. ${ }^{8}$, Bishop, K. ${ }^{9}$, Watchman, K., ${ }^{10}$ Janicki, M.P. ${ }^{11}$ and the Working Group of the International Summit on Intellectual Disability and Dementia

${ }^{1}$ University at Albany, New York USA

${ }^{2}$ Family Advocate, Eliot, Maine USA

${ }^{3}$ University of Minho, Braga, Portugal

${ }^{4}$ Trinity College Dublin, Dublin, Ireland

${ }^{5}$ Nurse Practitioner Consultant, Northampton, Massachusetts USA

${ }^{6}$ Reena Foundation, Toronto, Ontario Canada

${ }^{7}$ American Academy of Developmental Medicine and Dentistry, Lumberton, New Jersey USA

${ }^{8}$ Catalan Foundation for Down Syndrome, Barcelona, Spain

${ }^{9}$ Geriatrics Consultant, Lee Center, New York USA

${ }^{10}$ University of Stirling, Scotland

${ }^{11}$ University of Illinois at Chicago, Illinois USA

Address for Corresponding Author:

Philip McCallion, Ph.D.

Center for Excellence in Aging Services

RI 383

University at Albany

Albany NY 12222 USA

Email: pmccallion@albany.edu

Telephone: +1 $518442-5347$

\footnotetext{
${ }^{1}$ This consensus statement was developed as an output from the 2016 International Summit on Intellectual Disability and Dementia, held in Glasgow, Scotland, 13-14 October 2016, and hosted by the University of Stirling and University of the West of Scotland, funded by the RS MacDonald Trust, the Scottish Government, and Alzheimer Scotland. Collaborating sponsors included the National Task Group on Intellectual Disabilities and Dementia Practices (NTG) in the United States and the University of Illinois at Chicago. The Summit was composed of individuals and representatives of many international and national organizations with a stake in issues related to adults with intellectual disability affected by dementia. The contents of this statement were partially developed under a grant from the United States Department of Health and Human Services, Administration for Community Living (ACL), National Institute on Disability, Independent Living, and Rehabilitation Research (NIDILRR) Grant \# 90RT5020-03-00. However, those contents do not necessarily represent the policy of the US Department of Health and Human Services, nor the endorsement by the US federal government. The opinions expressed represent those of the Summit participants and of the NTG.
} 


\begin{abstract}
Adults with intellectual disability are affected by dementia at equivalent and elevated rates; many surviving into advanced age. End-of-life care and support considerations come into play among these individuals when most are in the advanced stage of dementia. The International Summit on Intellectual Disability and Dementia's report on end-of-life care in advanced dementia provides a synthesis statement which encompasses defining the state of advanced dementia, proposing use of palliative care services (including hospice), and recommending special efforts for enabling advanced directives and advance care planning prior to the extensive progression of dementia. The Summit recommended that when aiding adults with advanced dementia, the following be undertaken: integrative efforts between intellectual disability and palliative care providers, specialized training for carers on end-of-life care and supports, and involvement of adults with intellectual disability early on in their advance care planning.
\end{abstract}

Key Words: dementia, end-of-life, intellectual disability, palliative, person-centered, statement 


\section{Introduction}

Dementia is a term that characterizes the progressive loss of brain function that occurs with certain neuropathological diseases or trauma, and ultimately results in death - usually from circulatory and respiratory (e.g., pneumonia) diseases (Brunnström \& Englund, 2009). Death can also result from dementia-related dysfunction, such as self-neglect, general wasting, malnutrition, and dehydration, which are contributory risks when an individual with dementia can no longer care for him or herself (Morrow, 2016). The time frame of progression to end-oflife in persons with dementia is complicated by a host of factors, including the person's overall general health and stamina, other chronic diseases or coincident conditions, the type of dementia, level of fragility and frailty, lack of or access to health services, and propensity for acute conditions, such as infections. Because of these risk factors, concerns related to end-of-life care are usually focused on persons with advanced dementia. Families or support services are confronted with managing a tension between supporting the person and enabling him or her to continue to lead a desired life, whilst at the same time supporting and preparing for ongoing decline in health and overall function and ultimately death. That death may be the direct result of the progression of dementia but may also be due to contributory co-morbid health conditions and/or be the direct result of those other health conditions.

An invitational meeting, the International Summit on Intellectual Disability and Dementia, was held in Glasgow, Scotland, on October 13-14, 2016; its attendees representing organizations from 12 countries, were charged with examining a number of dementia-related issues affecting people with intellectual disability. The Summit's outcomes included a series of consensus statements and reports, one of which examined the consequences of end-of-life in advanced dementia

The Summit believes that what differentiates end-of-life issues among adults with intellectual disability and dementia from their other end-of life issues are three primary features: (1) pending death as a function of progression to the advanced stage of dementia (with the expectation that ceasing of primary functions will lead to organ failure or associated lifethreatening illness, such as pneumonia); (2) pending death associated with a life-threatening coincident condition (e.g., cancer, heart failure) irrespective of stage of dementia, and (3) pending death due to trauma or injury unassociated with dementia. The additional consideration is 
involvement in care decision-making; when advanced dementia is present there is an inability to participate in decision-making due to severe cognitive impairment.

The challenge to define what is meant by the end-of-life phase for all individuals with advanced dementia is particularly more difficult among individuals with an intellectual disability. The statement seeks to address this challenge and to offer care guidance.

\section{Statements and Recommendations}

Advanced dementia. Late or advanced stage of dementia presents with gross debilitation and terminates with death. The features of advanced dementia include profound memory deficits (e.g., inability to recognize family), extremely limited verbal communication, loss of ambulatory abilities, the inability to perform activities of daily living, loss of muscular control, seizures (usually in Down syndrome), and urinary and fecal incontinence (McCarron et al., 2017; Mitchell, 2015). The frequency and severity of seizures is an area where advanced dementia is different for people with an intellectual disability; but, as advanced dementia in people with intellectual disability requires more detailed description and documentation, it is addressed in another Summit paper (see McCarron et al., 2017)

Needs at end-of-life. Defining need and nature of care is often challenging as each dying adult with advanced dementia will present with different features and have different levels of ability and preparedness to participate in his or her care planning (Jokinen et al., 2013). Defining the end stage of life and its likely duration is difficult, but it is particularly difficult with persons with intellectual disability and advanced dementia when the scales recommended for use in the general population (Sheehan, 2012), such as the Global Deterioration Scale (GDS; Reisberg et $a l ., 1982$ ) or its successor, the Functional Assessment Staging Tool (FAST; see stage 7; Reisberg, 1988), tend to define the last months of life (and in some countries base eligibility for hospice and other palliative care approaches) in terms of inabilities for affected persons to dress themselves, walk without assistance, bathe properly without assistance, maintain continence of urine and stool, and speak or meaningfully communicate. A complication is that many adults with severe forms of intellectual disability may effectively qualify for stage 7 , but not have dementia or be dying. A broader consideration of status is needed and we recognize the 
importance of clinically defining when this, often extended, advanced stage of dementia is reached. Clinical practice supports the supposition that individuals with intellectual disability can be diagnosed with advanced dementia if the FAST (or equivalent) criteria are met AND are accompanied by acute medical complications such as multiple hospitalizations, recurrent infections (e.g., urinary tract infections, blood infections), aspiration pneumonia (resulting from choking on food or fluids), pressure sores on the skin, and/or refusal to eat. Together these may signal that remaining life expectancy is of fewer than six months and thus in turn it means there is a need for specialized end-of-life care.

Palliative and hospice care. Palliative and hospice care are often considered to be synonymous with end-of-life care. The WHO defines palliative care as "an approach that improves the quality of life of patients and their families facing the problem associated with life-threatening illness, through the prevention and relief of suffering by means of early identification and impeccable assessment and treatment of pain and other problems, physical, psychosocial and spiritual" (WHO, 2016). Hospice care involves a team-oriented approach to expert medical care, pain management, and emotional and spiritual support expressly tailored to an individual's needs and wishes. It is the belief of the Summit group that palliative and hospice care definitions may not always completely capture the experience, care and challenges of end-of-life care for people with an intellectual disability.

The Summit recognizes that palliative care can address needs before the final months, but that current conceptualizations must be expanded and be expressed more in terms of personcentered and relationship-centered care. This means that palliative care among adults with intellectual disability must better reflect the philosophies underpinning care and expertise inherent in both intellectual disability and specialist palliative care services (McCallion et al., 2012). Knowledge and specific skills for workers in intellectual disability should be enhanced, particularly in managing symptoms such as pain, constipation, dyspnea and fevers, managing nutrition and hydration, and assessing pain/distress. Staff in specialist palliative care, who can offer help with symptom management and be an external source of support that may be vital to dying adults, would also benefit from learning about practices used by staff in intellectual disabilities services and from families. Here areas for training include better management of communication with the person with intellectual disability; facilitating grief and loss, and 
addressing disenfranchised grief among staff and peers (Nolan et al., 2006). In the end stage of dementia-related care, providers, family, intellectual disability services, and palliative and hospice providers must effectively work together to address gaps in service provision and offer expertise/training. Such cooperation should include attention to the skill mix among existing staff resources, inclusion of intellectual disability-specific expertise and the opportunity to build upon values, needs, and preferences already expressed in existing person-centered plans for the person with intellectual disability. This collective work is increasingly relevant, as more persons present with advanced dementia and wish to remain in their place of care in their last months of life - a desire their carers often share (McCallion et al., 2012).

Person-centered, palliative, and end-of-life care should be about living and dying well when confronted with advanced dementia. Quality dementia-capable care should incorporate elements of a palliative care approach intent on improving the quality of life of adults and their families, and the prevention and relief of suffering through early identification, assessment and treatment of pain, as well as physical, and psychosocial and spiritual concerns (Kerr, Cunningham \& Wilkinson, 2006, 2008). It must also include responses that reflect the person's preferences and his or her cultural and ethnic values.

The Summit concurs with the recommendations of the European Association for Palliative Care Taskforce for People with Intellectual Disabilities (European Association for Palliative Care, 2015; Tuffrey-Wijne et al., 2016) with respect to equity of access for people with intellectual disability to palliative care services and supports, and agree that for people with intellectual disability and advanced dementia this will only occur if there is (a) recognition of their differing communication needs; (b) appreciation of health and social care professionals' alertness to signs and symptoms of serious illness, end of life and the dying phase; (c) inclusion of people who matter to the person with intellectual disability in symptom management, education and training; (d) support for families, carers and peers; (e) collaboration between intellectual disability services, hospital-based primary care, and palliative care and hospice care professionals with family and peer carers and the person with advanced dementia; and (f) hospice care in- and out-of-home which offers supportive care focused on comfort and quality of life in the final phase of dementia. 
Place of Care. As with other persons in general, place of care at end-of-life must include offering person-centered and palliative and hospice support in a range of living and care options, from own or family home, domiciliary, group home, specialized disability services settings, and/or (when appropriate) facilities with 24-hour skilled nursing supports. In advanced dementia, there should also be the opportunity to receive day supports, as well as respite, family support, and supportive interventions in their home (Hanson et al., 2016; Nickle \& McCallion, 2005). Dementia-related supports and sensory-based interventions that are designed to maintain quality of life and valued relationships should be offered and constructed along person-centered planning principles.

Planning for End of Life. The Summit believes that similar to what is being encouraged for all persons with dementia and other terminal conditions a discussion of goals and expectations around end-of-life care should start early, preferably before onset of the disease or neuropathology and only if the person and their carers desire. Advance planning gives the individual opportunities to indicate choices about specific treatments, such as resuscitation, preferred intensity for medical interventions or preferences for artificially administered nutrition (e.g., feeding tubes) and hydration. Carers (both family and staff) are accustomed to advocating for active treatment. The decision in advanced dementia, and indeed in other end stage diseases, to possibly forgo resuscitation, hospital admission, antibiotic use, feeding tubes, and other life prolonging efforts, can often be confusing and distressing for carers (McCarron \& McCallion, 2007). Waiting until dementia is present to discuss end-of-life and assuming that any decisions solely need to be discussed once it presents only adds to the burden of any diagnosis. Waiting until the last days of life to begin these decisions is too limiting and too late. There are also a number of emotional and personal aspects to consider in end-of-life care. End-of-life is also about maintaining and celebrating the individual and his or her life history, and end-of-life care is also about supporting those who remain after death by commemorating a life which had meaning. Persons affected by dementia deserve no less. Positioning end-of-life within an understanding of the person's life history and lifelong person-centered planning around their values and preferences facilitates this discussion. However, it is acknowledged that such discussions in many cases have not yet occurred because persons with an intellectual disability, families, and paid carers lack information on decision points in the transition from active to palliative care, while remaining committed to preserving desired quality of life. This lack of 
information needs to be addressed collectively by the intellectual disability services and the palliative/hospice care systems.

Advance Care Planning. The Summit recognizes that planning (whether formal organized or informal) is a dynamic and continuing process of considering and documenting people's wants, preferences, and choices prior to diagnosis and expressed preferences should be considered as an individual's condition changes (Bischoff et al., 2013; Wiese et al., 2013). Legal frameworks in different jurisdictions offer varying guidance on completing oral or written advance directives (such as, the appointment of a health care proxy, or an equivalent). There are similar issues around definition of and capacity for consent. This applies not only to consent, but also to the legal status of advance care planning, the implications of determining capacity among adults with more severe levels of intellectual disability, ability to understand and participate in planning and understanding and consenting to palliative/hospice services.

Exploring, through person-centered planning, a person's values and preferences throughout life will offer the best guide to the person's wishes for care and supports in his or her final days. The Summit recommends involving people with intellectual disabilities in decisions about their end-of-life care, drawing upon statements of values in their person-centered plans, where available. Practitioners and family are encouraged to ascertain and honor these desires. In jurisdictions where advance planning is encouraged and guided by legal and/or accepted practice frameworks, protocols must be in place to ensure that people with an intellectual disability have the same opportunities to express their desires in a formal manner (see for example, Stein, 2007). There must also be educational materials and interventions to support both the individual with intellectual disability and their carers, family, peers, and staff.

Educational and Professional Materials. The Summit believes that staff and other personnel working with older adults should be provided with training in key issues related to end-of-life care and the opportunity to examine their own values and perspectives on dying and death. Provider organizations must develop, distribute, and implement educational materials for carers and others related to advanced dementia and end-of-life. There are curricula and content resources available (See, for example, Fahey-McCarthy et al., 2008, 2009; Gaventa, 2012). Workers can also benefit from formal practice guidelines and related consensus documents on standards of care and generally accepted practices (see Janicki \& Keller, 2015; Jokenin et al., 
2013). Governmental and educational groups must encourage the needed cross-organizational efforts.

\section{Commentary}

The Summit recognized that there are unique considerations when progressive dementia is a factor in the last days of life for a person with an intellectual disability. End-of-life care and support in general is better undertaken when there are tools and training available, but that is not enough. There is a challenge for mainstream services, including palliative/hospice services, to effectively include adults with intellectual disability and their families and other carers in care decision-making and being easily able to access and receive needed services. Further, the Summit recognizes that the focus cannot be on primary medically-oriented end-of-life care. Adults with intellectual disability affected by dementia bring their own life stories, their own relationships, and their own service history with them to the end of life. In advanced dementia continuing the connection with that history and hearing the voice of the person is critical. Endof-life is not simply about procedures and interventions, palliative or hospice; it must be about the person living his or her last days as desired and their family, friends, and paid carers completing that journey with them. Person-centered approaches that have improved the lives of so many people with intellectual disability must be continued to the end of life. Rather than new and unique approaches, end-of-life care must build upon and benefit from this history and fulfil the realization of each person's values and preferences.

To further understanding and capability in supporting adults at the end-of-life with advanced dementia, the Summit recommends that:

1. Intellectual disability, dementia, and palliative care organizations and associations coalesce and create a universal practice guideline on end-stage care and support practices for persons with advanced dementia

2. Training curricula developed and utilized by intellectual disability, dementia and palliative care organizations and associations to address advanced dementia and intellectual disability must emphasize relationship and person-centered care approaches. 
3. In taking a lifespan approach to care of people with intellectual disability support organizations must include strategies for altering or increasing supports and decision-making to address changing care needs with dementia progression, including as the individual approaches end-of-life.

4. Care and support organizations must commit to infusing in their staff, family support, and services delivery structures the belief that the essence of each person remains throughout the progression of dementia.

\section{References}

Bischoff, K.E, Sudore, R., Miao, Y., Boscardin, W.J., \& Smith, A.K. (2013). Advance care planning and the quality of end-of-life care among older adults. Journal of the American Geriatrics Society, 61(2), 209-214. DOI:10.1111/jgs.12105

Brunnström, H.R., \& Englund, E.M. (2009). Cause of death in patients with dementia disorders. European Journal of Neurology, 16(4), 488-492. DOI: 10.1111/j.1468-1331.2008.02503.x

European Association for Palliative Care. (2015). Consensus norms for palliative care of people with intellectual disabilities in Europe: EAPC White Paper. Accessed from: www.learningdisabilityanddementia.org/uploads/1/1/5/8/11581920/eapc-white-paper-id_fullversion_april-2015.pdf

Fahey-McCarthy, E., McCallion, P., Connaire, K., McCarron, M. (2008). Supporting persons with intellectual disability and advanced dementia: Fusing the horizons of care. An introductory education and training programme. Trainer's manual. Dublin, Ireland: Trinity College Dublin. Accessed from: https://nursing-midwifery.tcd.ie/assets/publications/pdf/fusing-horizons-ofcare.pdf.

Fahey-McCarthy, E., McCarron, M., Connaire, K., \& McCallion, P. (2009). Developing an education intervention for staff supporting persons with an intellectual disability and advanced dementia. Journal of Policy and Practice in Intellectual Disability, 6(4), 267-275. 
Hanson, E., Hellstrom, A., Sandvide, A., Jackson, G.A., MacRae, R., Waugh, A., Abreu, W. \& Tolson, D. (2016). The extended palliative phase of dementia - An integrative literature review. Dementia. Epub ahead of print. DOI: 10.1177/1471301216659797

Gaventa, W. (2012). Resources in developmental disabilities and coping with grief, death and dying. New Brunswick, New Jersey: Elizabeth M. Boggs Center on Developmental Disabilities, University of Medicine \& Dentistry of New Jersey. Accessed from http://rwjms.rutgers.edu/departments_institutes/boggscenter/documents/EndofLifeResources120 13.pdf.

Janicki, M.P., \& Keller, S.M. (2015). Why do we need national guidelines for adults with intellectual disability and dementia? Editorial. Alzheimer's \& Dementia: Diagnosis, Assessment \& Disease Monitoring, 1(3), 325-327.

Jokinen, N., Janicki, M.P., Keller, S.M., McCallion, P., Force, L.T., and the National Task Group on Intellectual Disabilities and Dementia Practices. (2013). Guidelines for structuring community care and supports for people with intellectual disabilities affected by dementia. Journal of Policy and Practice in Intellectual Disabilities, 10(1), 1-28.

Kerr, D. Cunningham, C., \& Wilkinson, H. (2006). Pain management for older people with learning difficulties and dementia. Joseph Roundtree Foundation. Accessed from: https://www.jrf.org.uk/report/pain-management-older-people-learning-difficulties-and-dementia Kerr, D., Cunningham, C., \& Wilkinson, H. (2008). Do you recognise pain in someone with a learning difficulty and dementia? A guide for GPs. Joseph Roundtree Foundation. Accessed from: https://www.jrf.org.uk/sites/default/files/jrf/migrated/files/pain-dementia-factsheet.pdf Kirkendall, A., Linton, K., \& Farris, S. (2016). Intellectual disabilities and decision making at end of life: A literature review. Journal of Applied Research in Intellectual Disabilities. Advance online publication. DOI: 10.1111/jar.12270

McCarron, M., \& McCallion, P. (2007). End-of-life care challenges for persons with intellectual disability and dementia: making decisions about tube feeding. Intellectual and Developmental Disabilities, 45(2), 128-131. 
McCarron, M., Stemp, S., Coppus, T., Fortea, J., McCallion, P., Reilly, E., Watchman, K., Janicki, M.P. (2017). Consensus statement of the international summit on intellectual disability and dementia related to delivering quality care for adults with intellectual disability and advanced dementia. Accessed from: www.aadmd.org/ntg/summit and http://www.learningdisabilityanddementia.org/id-dementia-summit.html.

McCallion, P., McCarron, M., Fahey-McCarthy, E., \& Connaire, K. (2012). Meeting the end of life needs of older adults with intellectual disabilities. In C. Chang \& A. Johnson, (Eds.), Contemporary and Innovative Practice in Palliative Care. Intech. Accessed from: http://www.intechopen.com/books/contemporary-and-innovative-practice-in-palliativecare/meeting-the-end-of-life-needs-of-older-adults-with-intellectual-disabilities

Mitchell, S.L. (2015). Advanced dementia. New England Journal of Medicine, 372, 2533-2540. DOI: 10.1056/NEJMcp1412652

Morrow, A., (2016). What is it like to die of dementia? Very Well. Accessed from https://www.verywell.com/what-is-it-like-to-die-of-dementia-1132331

Nickle, T., \& McCallion, P. (2005). Redesigning day programmes. The Frontline of Learning Disability, 64(Winter), 27-28.

Nolan, M.R., Brown, J., Davies, S., Nolan, J., \& Keady, J. (2006). The Senses Framework: improving care for older people through a relationship-centred approach. Getting Research into Practice (GRiP) Report No 2. Project Report. Sheffield, United Kingdom: University of Sheffield. Accessed from: http://shura.shu.ac.uk/280/1/PDF_Senses_Framework_Report.pdf Reisberg, B. (1988). Functional assessment staging (FAST). Psychopharmacological Bulletin. 24(4), 653-659.

Reisberg, B., Ferris, S.H., de Leon, M.J., \& Crook, T. (1982). The global deterioration scale for assessment of primary degenerative dementia. American Journal of Psychiatry, 139(9),11361139.

Sheenan, B. (2012). Assessment scales in dementia. Therapeutic Advances in Neurological Disorders, 5(6), 349-358. 
Stein, G.L. (2007). Advance directives and advance care planning for people with intellectual and physical disabilities. Washington, DC: Office of the Assistant Secretary for Planning and Evaluation. Accessed from: https://aspe.hhs.gov/basic-report/advance-directives-and-advancecare-planning-people-intellectual-and-physical-disabilities

Tuffrey-Wijne, I., Wicki, M., Heslop, P. McCarron, M., Todd, S., Oliver, D., ... Curfs, L. (2016). Developing research priorities for palliative care of people with intellectual disabilities in Europe: a consultation process using nominal group technique. BMC Palliative Care, 15, 36c.

DOI: $10.1186 / \mathrm{s} 12904-016-0108-5$.

WHO. (2016). WHO definition of palliative care. Geneva, Switzerland: Author. Accessed from: http://www.who.int/cancer/palliative/definition/en/

Wiese, M., Dew, A., Stancliffe, R.J., Howarth, G., \& Balandin, S. (2013). "'If and when?":

Experiences of community living staff engaging older people with intellectual disability to know about dying." Journal of Intellectual Disability Research, 57(10), 980-992. DOI:

10.1111/j.1365-2788.2012.01593.x 